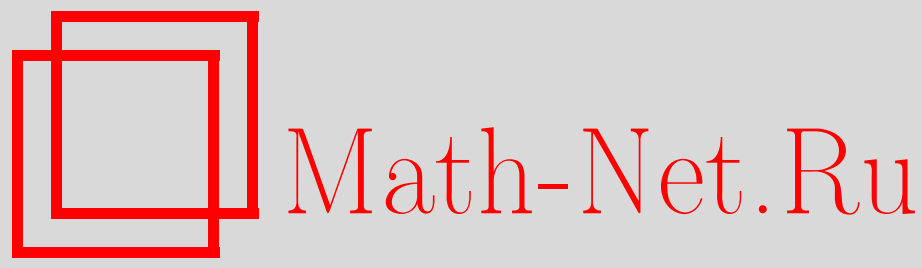

В. В. Соколов, С. Я. Старцев, Симметрии нелинейных гиперболических систем типа цепочек Тоды, ТМФ, 2008, том 155, номер 2, 344-355

DOI: https://doi.org/10.4213/tmf6216

Использование Общероссийского математического портала Math-Net.Ru подразумевает, что вы прочитали и согласны с пользовательским соглашением http://www . mathnet.ru/rus/agreement

Параметры загрузки:

IP : 54.224 .135 .184

26 апреля 2023 г., $07: 33: 42$

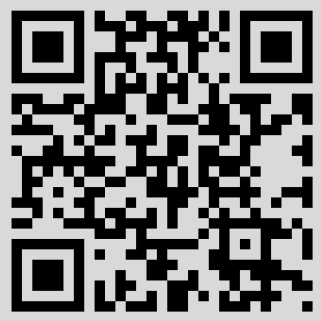




\title{
ФИЗИКА
}

Том 155, № 2

май, 2008

2008 г.

В. В. Соколов*, С. Я. Старцев ${ }^{\dagger}$

\section{СИММЕТРИИ НЕЛИНЕЙНЫХ ГИПЕРБОЛИЧЕСКИХ СИСТЕМ ТИПА ЦЕПОЧЕК ТОДЫ}

\begin{abstract}
Рассмотрены гиперболические системы уравнений, обладающие полными наборами интегралов по обоим характеристикам. Наиболее известным примером моделей такого типа являются двумеризованые открытые цепочки Тоды. Для систем, обладающих интегралами, построен дифференциальный оператор, переводящий интегралы в симметрии. Тем самым для систем указанного типа доказано существование высших симметрий, зависящих от произвольных функций.
\end{abstract}

Ключевые слова: уравнение Лиувилля, цепочка Тоды, интегралы, высшие симметрии, гиперболические системы уравнений в частных производных, теорема Нётер.

\section{1. ВВЕДЕНИЕ}

Простейшими интегрируемыми уравнениями вида

$$
u_{x y}=F\left(x, y, u, u_{x}, u_{y}\right)
$$

являются уравнения, похожие по своим свойствам на уравнение Лиувилля $u_{x y}=e^{u}$. Одним из таких свойств является наличие нетривиальных $x$ - и $y$-интегралов (таких функций, зависящих от $x, y, u$ и производных $u$, что их полные производные соответственно по $y$ и $x$ равны нулю в силу уравнения). В случае уравнения Лиувилля интегралы минимального порядка задаются формулами

$$
w=u_{x x}-\frac{u_{x}^{2}}{2}, \quad \bar{w}=u_{y y}-\frac{u_{y}^{2}}{2} .
$$

Действительно, нетрудно проверить, что $D_{x}(\bar{w})=0$ и $D_{y}(w)=0$, где через $D_{x}$ и $D_{y}$ обозначены полные производные в силу уравнения Лиувилля по $x$ и $y$ соответственно. Помимо существования нетривиальных интегралов уравнение Лиувилля обладает еще одним важным для нас свойством - в работе [1] было показано, что для любой функции $g$, зависящей от конечного числа аргументов, формула

$$
s=\left(D_{x}+u_{x}\right)\left(g\left(x, w, D_{x}(w), D_{x}^{2}(w), \ldots\right)\right)
$$

* Институт теоретической физики им. Л. Д. Ландау, г. Черноголовка, Московская обл., Россия. E-mail: sokolov@itp.ac.ru

${ }^{\dagger}$ Институт математики с ВЦ УНЦ РАН, Уфа, Россия. E-mail: startsev@anrb.ru 
задает решение линеаризованного уравнения Лиувилля $D_{x} D_{y}(s)=e^{u} s$, т.е. $s$ является высшей инфинитезимальной симметрией уравнения Лиувилля. Эта симметрия зависит только от производных функции $u$ по переменной $x$. Аналогичная формула имеется и для $y$-симметрий.

Уравнение (1) допускает симметрии сколь угодно высокого порядка, если у этого уравнения существуют нетривиальные интегралы по каждой из характеристик. При этом структура симметрий остается той же самой. Меняется только дифференциальный оператор $S$, порождающий симметрии и в случае уравнения Лиувилля имеющий вид $S=D_{x}+u_{x}$. Этот факт был доказан в работе [2] с использованием каскадного метода интегрирования Лапласа (см., например, [3]). Кроме того, в работе [2] приведена явная формула для этого оператора в терминах инвариантов Лапласа линеаризации уравнения (1).

Обобщением уравнения Лиувилля на случай систем вида

$$
u_{x y}^{i}=F^{i}\left(x, y, u, u_{x}, u_{y}\right), \quad i=1,2, \ldots, n,
$$

являются открытые цепочки Тоды

$$
u_{x y}^{i}=\exp \left(\sum_{j=1}^{n} A_{i j} u^{j}\right), \quad i=1,2, \ldots, n,
$$

где $A_{i j}$ - элементы матрицы Картана простой алгебры Ли (см. [4], [5]). Согласно [6], [7] эти системы обладают полным набором $x$ - и $y$-интегралов. Например, для системы из двух уравнений

$$
p_{x y}=e^{2 p-q}, \quad q_{x y}=e^{2 q-p}
$$

два функционально независимых $x$-интеграла минимального порядка имеют вид

$$
\begin{aligned}
& w_{1}=p_{x x}+q_{x x}-p_{x}^{2}-q_{x}^{2}+p_{x} q_{x}, \\
& w_{2}=p_{x x x}-p_{x}\left(2 p_{x x}-q_{x x}\right)+q_{x} p_{x}^{2}-p_{x} q_{x}^{2} .
\end{aligned}
$$

Симметрии для цепочек Тоды ранга 2 были найдены в [8], [9] с помощью так называемого метода спуска. В случае системы (3) они имеют вид

$$
\begin{aligned}
\left(\begin{array}{l}
s_{1} \\
s_{2}
\end{array}\right)= & \left(D_{x}+\left(\begin{array}{ll}
3 p_{x}-2 q_{x} & 2 q_{x}-2 p_{x} \\
2 p_{x}-2 q_{x} & 3 q_{x}-2 p_{x}
\end{array}\right)\right) \circ \\
& \circ\left(D_{x}+\left(\begin{array}{ll}
2 q_{x}-p_{x} & p_{x}-2 q_{x} \\
q_{x}-2 p_{x} & 2 p_{x}-q_{x}
\end{array}\right)\right)\left(\begin{array}{l}
f_{1} \\
f_{2}
\end{array}\right),
\end{aligned}
$$

где $f_{1}$ и $f_{2}$ - произвольные $x$-интегралы (т.е. любые функции, зависящие от конечного числа аргументов из набора $\left.x, w_{1}, w_{2}, D_{x}\left(w_{1}\right), D_{x}\left(w_{2}\right), D_{x}^{2}\left(w_{1}\right), D_{x}^{2}\left(w_{2}\right), \ldots\right)$.

В настоящей работе доказано, что любая система (2), обладающая полным набором $w_{1}, \ldots, w_{n}, \bar{w}_{1} \ldots, \bar{w}_{n}$ из $x$ - и $y$-интегралов, имеет симметрии вида

$$
\mathbf{s}=\sum_{i=0}^{r} \alpha_{i} D_{x}^{r-i}(\mathbf{f})
$$


где $\mathbf{f}$ - произвольная вектор-функция, зависящая от $x$-интегралов $w_{1}, \ldots, w_{n}$ и их полных производных по переменной $x$. Для лагранжевых систем существование симметрий указанного вида можно доказать с помощью теоремы Нётер (см. [10], [11]). В общем случае было бы естественным попытаться обобщить доказательство соответствующего результата из работы [9], посвященной скалярному случаю. Однако для систем использование метода инвариантов Лапласа наталкивается на серьезные трудности. Наш способ построения дифференциального оператора

$$
S=\sum_{i=0}^{r} \alpha_{i} D_{x}^{r-i}
$$

основан на другом подходе. Конструктивность этого подхода мы демонстрируем на методическом примере хорошо известной интегрируемой системы

$$
p_{x y}=\frac{q p_{x} p_{y}}{p q+c}, \quad q_{x y}=\frac{p q_{x} q_{y}}{p q+c}
$$

где $c$ - отличная от нуля константа.

\section{2. ОБОЗНАЧЕНИЯ, ОПРЕДЕЛЕНИЯ И ИЗВЕСТНЫЕ ФАКТЫ}

Перепишем систему (2) в векторной форме

$$
\vec{u}_{x y}=\vec{F}\left(x, y, \vec{u}, \vec{u}_{x}, \vec{u}_{y}\right),
$$

где $\vec{u}=\left(u^{1}, u^{2}, \ldots, u^{n}\right)^{\mathrm{T}}, \vec{F}=\left(F^{1}, F^{2}, \ldots, F^{n}\right)^{\mathrm{T}}$. Так как смешанные производные $\vec{u}$ мы можем исключить в силу системы (2), все локальные объекты (симметрии, $x$ - и $y$-интегралы, коэффициенты оператора $S$ и других дифференциальных операторов) являются функциями, зависящими от конечного числа независимых переменных $x$, $y, \vec{u}, \vec{u}_{i}=\partial^{i} \vec{u} / \partial x^{i}, \vec{v}_{i}=\partial^{i} \vec{u} / \partial y^{i}$. Обозначим кольцо таких функций через $\mathcal{F}$. Будем говорить, что функция $f \in \mathcal{F}$ имеет порядок $(k, m)$, если $\vec{v}_{k}$ и $\vec{u}_{m}$ являются старшими из частных производных $\vec{u}$, от которых зависит функция $f$. Если функция $f$ имеет порядок $(k, m)$, мы будем писать $\operatorname{ord}_{y}(f)=k, \operatorname{ord}_{x}(f)=m$.

На множестве $\mathcal{F}$ действуют операторы полных производных $D_{x}$ и $D_{y}$ в силу системы (2):

$$
\begin{aligned}
& D_{x}(g)=\frac{\partial g}{\partial x}+\frac{\partial g}{\partial \vec{u}} \vec{u}_{1}+\sum_{i=1}^{\infty}\left(\frac{\partial g}{\partial \vec{u}_{i}} \vec{u}_{i+1}+\frac{\partial g}{\partial \vec{v}_{i}} D_{y}^{i-1}(\vec{F})\right) \\
& D_{y}(g)=\frac{\partial g}{\partial y}+\frac{\partial g}{\partial \vec{u}} \vec{v}_{1}+\sum_{i=1}^{\infty}\left(\frac{\partial g}{\partial \vec{v}_{i}} \vec{v}_{i+1}+\frac{\partial g}{\partial \vec{u}_{i}} D_{x}^{i-1}(\vec{F})\right) .
\end{aligned}
$$

Здесь через $g_{z}=\partial g / \partial z$, где $g$ - скалярная функция, $z$ - вектор $\left(z^{1}, z^{2}, \ldots, z^{n}\right)^{\mathrm{T}}$, обозначена строка $\left(\partial g / \partial z^{1}, \partial g / \partial z^{2}, \ldots, \partial g / \partial z^{n}\right)$. На векторах и матрицах действие $D_{x}$ и $D_{y}$ определяется покомпонентно. Легко проверить, что операторы $D_{x}$ и $D_{y}$ коммутируют между собой.

Пользуясь симметрией формулы (2) относительно замены $x \leftrightarrow y$, далее мы будем приводить лишь одно из двух “симметричных" определений и утверждений. 
ОПРЕДЕЛЕНИЕ 1 . Функцию $w \in \mathcal{F}$ будем называть $x$-интегралом системы $(2)$, если $D_{y}(w)=0$. Если $w$ зависит только от $x$, то $w$ будем называть тривиалъным х-интегралом.

Нетрудно видеть, что $x$-интеграл не может зависеть от переменных $\vec{v}_{i}$. Порядок старшей из частных производных $\vec{u}_{i}$, от которых интеграл существенно зависит, будем называть порядком интеграла.

Пусть $w^{1}, w^{2}, \ldots, w^{r}-x$-интегралы системы (2) порядка $p_{1}, p_{2}, \ldots, p_{r}$ соответственно. Будем говорить, что эти интегралы независимы в главном, если матрица размера $r \times n$, составленная из строк $\partial w^{1} / \partial \vec{u}_{p_{1}}, \partial w^{2} / \partial \vec{u}_{p_{2}}, \ldots, \partial w^{r} / \partial \vec{u}_{p_{r}}$ имеет ранг $r$. Ясно, что система (2) не может иметь более чем $n$ интегралов, независимых в главном.

ОПРЕДЕЛЕНиЕ 2. Вектор-функция $f$ с компонентами из $\mathcal{F}$ называется локалъной инфинитезимальной симметрией системы (2), если выполнено соотношение $L(f)=0$, где

$$
L=D_{x} D_{y}-\vec{F}_{\vec{u}_{x}} D_{x}-\vec{F}_{\vec{u}_{y}} D_{y}-\vec{F}_{\vec{u}} .
$$

Поясним, что для любого вектора $z=\left(z^{1}, z^{2}, \ldots, z^{n}\right)^{\mathrm{T}}$ и вектор-функции $G=$ $\left(G^{1}, G^{2}, \ldots, G^{n}\right)^{\mathrm{T}}$ через $G_{z}$ обозначается матрица со строками $G_{z}^{1}, \ldots, G_{z}^{n}$. Дифференциальный оператор (5) называется оператором линеаризации системь (2).

Для любой функции $g \in \mathcal{F}$ определим дифференциальный оператор

$$
g_{*}=\frac{\partial g}{\partial \vec{u}}+\sum_{i=1}^{\infty}\left(\frac{\partial g}{\partial \vec{v}_{i}} D_{y}^{i}+\frac{\partial g}{\partial \vec{u}_{i}} D_{x}^{i}\right) .
$$

Этот оператор будем называть линеаризацией функции $g$. Линеаризация векторфункции $g$ определяется этой же формулой.

Нам потребуется следующая

Лемма 1. Пусть все компоненты вектора $W$ являются $x$-интегралами порядка $k$ для системы (2). Тогда найдется дифференциалъный оператор $P=\sum_{i=0}^{k-1} \alpha_{i} D_{x}^{i}$, где $\alpha_{i}$ - некоторые матрицы с элементами из $\mathcal{F}$, такой, что

$$
D_{y} \circ W_{*}=P \circ L
$$

где L задается формулой (5).

ДокАЗАТЕЛЬство. Покажем, что для любого вектора $g$ можно найти дифференциальный оператор $P=\sum_{i=0}^{k-1} \alpha_{i} D_{x}^{i}, k=\operatorname{ord}_{x}(g)$, такой, что

$$
D_{y} \circ g_{*}=\left(D_{y}(g)\right)_{*}+P \circ L,
$$

где $L$ - оператор линеаризации системы (2). Действительно, нетрудно проверить, что

$$
\begin{gathered}
D_{y} \circ g_{*}=D_{y}\left(g_{\vec{u}}\right)+\sum_{i=1}^{\infty}\left(D_{y}\left(g_{\vec{v}_{i}}\right) D_{y}^{i}+D_{y}\left(g_{\vec{u}_{i}}\right) D_{x}^{i}\right)+ \\
+g_{\vec{u}} D_{y}+\sum_{i=1}^{\infty}\left(g_{\vec{v}_{i}} D_{y}^{i+1}+g_{\vec{u}_{i}} D_{y} D_{x}^{i}\right)
\end{gathered}
$$




$$
\begin{array}{r}
\left(D_{y}(g)\right)_{*}=D_{y}\left(g_{\vec{u}}\right)+\sum_{i=1}^{\infty}\left(D_{y}\left(g_{\vec{v}_{i}}\right) D_{y}^{i}+D_{y}\left(g_{\vec{u}_{i}}\right) D_{x}^{i}\right)+ \\
+g_{\vec{u}} D_{y}+\sum_{i=1}^{\infty}\left(g_{\vec{v}_{i}} D_{y}^{i+1}+g_{\vec{u}_{i}}\left[D_{x}^{i-1}(\vec{F})\right]_{*}\right),
\end{array}
$$

и, следовательно,

$$
\left(D_{y}(g)\right)_{*}=D_{y} \circ g_{*}+\sum_{i=1}^{k} g_{\vec{u}_{i}}\left(\left[D_{x}^{i-1}(\vec{F})\right]_{*}-D_{y} D_{x}^{i}\right) .
$$

Повторяя аналогичные рассуждения для дифференцирования $D_{x}$, получим, что для любого вектора $q$ такого, что $\operatorname{ord}_{y}(q) \leqslant 1$, верна формула

$$
\left(D_{x}(q)\right)_{*}=D_{x} \circ q_{*}-q_{\vec{u}_{y}} L .
$$

Учитывая, что $\operatorname{ord}_{y}\left(D_{x}^{i}(\vec{F})\right) \leqslant 1$ для любого $i$, путем многократного применения формулы (9) получим

$$
\left(D_{x}^{i}(\vec{F})\right)_{*}=D_{x}^{i} \circ \vec{F}_{*}+\sum_{j=0}^{i-1} \gamma_{i j} D_{x}^{j} \circ L
$$

где $\gamma_{i j}$ - некоторые матрицы. Подстановка этого соотношения в (8) доказывает формулу (7). Из формулы (7) и соотношения $D_{y}(W)=0$ мы можем легко получить равенство (6). Лемма 1 доказана.

ЗАмечание. Равенства некоммутативных многочленов по $D_{x}$ и $D_{y}$ с коэффициентами из $\mathcal{F}$ (такие многочлены мы называем дифференциальными операторами), а также некоммутативных формальных рядов следует понимать следующим образом: два объекта считаются равными, если после приведения их к канонической форме $\sum c_{i j} D_{x}^{i} D_{y}^{j}$ коэффициенты $c_{i j}$ совпадают. Для записи операторов в каноническом виде мы пользуемся тем, что любую матрицу $\alpha$ можно "перебросить влево" с помощью формулы

$$
D_{x}^{m} \circ \alpha=\alpha D_{x}^{m}+\sum_{i=1}^{+\infty} C_{m}^{i} D_{x}^{i}(\alpha) D_{x}^{m-i}, \quad C_{m}^{i}=\frac{m(m-1) \ldots(m-i+1)}{i !},
$$

а также аналогичной формулы для $D_{y}$. Ясно, что при положительных $m$ сумма в правой части (10) конечна, так как $C_{m}^{i}=0$ при $i>m$. В этом случае $(10)-$ это просто формула Лейбница. При отрицательных $m$ формула (10) “доказывается" путем многократного применения интегрирования по частям.

\section{3. СИММЕТРИИ}

Пусть $w$ является $x$-интегралом порядка $p$. Тогда из того что $D_{x}$ и $D_{y}$ коммутируют, следует, что $D_{x}^{i}(w)$ также является $x$-интегралом для любого натурального $i$. При этом легко проверить, что

$$
\frac{\partial\left(D_{x}^{i}(w)\right)}{\partial \vec{u}_{p+i}}=\frac{\partial w}{\partial \vec{u}_{p}}
$$


Поэтому если у системы (2) существуют $n$ независимых в главном $x$-интегралов порядка не выше $k$, то имеются и $n$ независимых в главном $x$-интегралов порядка $k$. Для построенного из них $n$-мерного вектора $W$ имеем $\operatorname{det}\left(W_{\vec{u}_{k}}\right) \neq 0$. Все сказанное выше верно и для $y$-интегралов.

Основным результатом статьи является следующая

Теорема. Пусть для системы уравнений (2) найдутся $n$-мерный вектор $Z$, составленный из у-интегралов порядка $\mathrm{m}$, такой, что матрица $Z_{\vec{v}_{m}}$ невырожденна, и п-мерный вектор $W$, составленный из $x$-интегралов порядка $k$, такой, что матрица $W_{\vec{u}_{k}}$ невырожденна. Тогда найдутся матрицы $\alpha_{i}$ размера $n \times n$ с коэффичиентами из $\mathcal{F}$ такие, что $\operatorname{det}\left(\alpha_{0}\right) \neq 0$ и оператор

$$
S_{r}=\sum_{i=0}^{r} \alpha_{i} D_{x}^{r-i}
$$

переводит в симметрии системы (2) любой $n$-мерный вектор, составленный из $x$-интегралов. При этом верна оценка $r \leqslant(m-1) n$.

Для доказательства теоремы мы построим дифференциальные операторы $S_{r}$ и $Q_{r}=\sum_{i=0}^{r+1} \beta_{i} D_{x}^{r-i}$ такие, что

$$
L \circ S_{r}=Q_{r} \circ D_{y}
$$

Ясно, что тогда $S_{r}(g)$ является симметрией системы (2) для любого $n$-мерного вектора $g$ из $\operatorname{Ker} D_{y}$. Нетрудно проверить, что

$$
L \circ S_{r}=\left(D_{y}-\vec{F}_{\vec{u}_{x}}\right)\left(\alpha_{0}\right) D_{x}^{r+1}+\sum_{i=0}^{r-1}\left(L\left(\alpha_{i}\right)+\left(D_{y}-\vec{F}_{\vec{u}_{x}}\right)\left(\alpha_{i+1}\right)\right) D_{x}^{r-i}+L\left(\alpha_{r}\right)+\cdots,
$$

где многоточием обозначены слагаемые, содержащие ненулевую степень $D_{y}$. Поскольку правая часть (11) не содержит членов с нулевой степенью $D_{y}$, равенство (11) выполняется, если и только если $\alpha_{i}$ удовлетворяют цепочке соотношений

$$
\begin{aligned}
\left(D_{y}-\vec{F}_{\vec{u}_{x}}\right)\left(\alpha_{0}\right) & =0, \\
L\left(\alpha_{i}\right)+\left(D_{y}-\vec{F}_{\vec{u}_{x}}\right)\left(\alpha_{i+1}\right) & =0, \quad i=0, \ldots, r-1, \\
L\left(\alpha_{r}\right) & =0 .
\end{aligned}
$$

Таким образом, для доказательства теоремы нам достаточно показать, что найдутся матрицы $\alpha_{i}$, удовлетворяющие цепочке (12). Подчеркнем, что речь идет о разрешимости уравнений $(12)$ в классе матриц с коэффициентами из $\mathcal{F}$. Простейшее уравнение подобного сорта $D_{y}(w)=0$, определяющее $x$-интегралы, разрешимо далеко не для любой системы (2). Смысл теоремы состоит в том, что если уравнения $D_{y}(w)=0, D_{x}(\bar{w})=0$ имеют "максимальное" число решений в $\mathcal{F}$, то разрешима и система (12).

Докажем сначала несколько вспомогательных утверждений. 
ЛЕмма 2. Пусть для системы (2) найдется $n$-мерный вектор $W$, составленный из $x$-интегралов порядка $k$, такой, что матрица $W_{\vec{u}_{k}}$ невырожденна. Тогда найдутся последовательности матрии $\alpha_{i}, \beta_{i}$ размера $n \times n$ такие, что формальные ряды $S=\sum_{i=0}^{+\infty} \alpha_{i} D_{x}^{-i}$ и $Q=\sum_{i=-1}^{+\infty} \beta_{i} D_{x}^{-i}$ удовлетворяют соотношению

$$
L \circ S=Q \circ D_{y}
$$

причем $\operatorname{det}\left(\alpha_{0}\right) \neq 0$.

ДокАзАТЕльство. В силу леммы 1 выполняется соотношение

$$
D_{y} \circ W_{*}=P \circ L
$$

Тогда, взяв ряд $\left(W_{*}\right)^{-1}$ в качестве $S$ и ряд $P^{-1}$ в качестве $Q$, мы получим

$$
L \circ S=P^{-1} \circ P \circ L \circ S=P^{-1} \circ D_{y} \circ W_{*} \circ\left(W_{*}\right)^{-1}=Q \circ D_{y} .
$$

Заметим, что если ряды $S$ и $Q$ удовлетворяют (13), то и ряды $S \circ D_{x}^{j}, Q \circ D_{x}^{j}$ удовлетворяют (13) для любого $j$. Поэтому без нарушения общности мы можем считать, что старшая степень $D_{x}$ в $S$ может быть любой, какой нам удобно. Лемма 2 доказана.

Проиллюстрируем нахождение ряда $S$ на примере системы (4). У этой системы имеются два независимых в главном $x$-интеграла

$$
w_{1}=\frac{p_{x x}}{p_{x}}-\frac{p q_{x}}{p q+c}, \quad w_{2}=\frac{q_{x x}}{q_{x}}-\frac{q p_{x}}{p q+c} .
$$

Возьмем в качестве $W$ вектор $\left(w_{1}, w_{2}\right)^{\mathrm{T}}$. Тогда

$$
W_{*}=\gamma_{2} D_{x}^{2}+\gamma_{1} D_{x}+\gamma_{0},
$$

где матрицы $\gamma_{i}$ задаются формулами

$$
\gamma_{2}=\left(\begin{array}{cc}
\frac{1}{p_{x}} & 0 \\
0 & \frac{1}{q_{x}}
\end{array}\right), \quad \gamma_{1}=-\left(\begin{array}{cc}
\frac{p_{x x}}{p_{x}^{2}} & \frac{p}{p q+c} \\
\frac{q}{p q+c} & \frac{q_{x x}}{q_{x}^{2}}
\end{array}\right), \quad \gamma_{0}=\frac{1}{(p q+c)^{2}}\left(\begin{array}{cc}
-c q_{x} & p^{2} q_{x} \\
q^{2} p_{x} & -c p_{x}
\end{array}\right) .
$$

Введем дифференциальный оператор $R=2 \gamma_{2} D_{x}+\gamma_{1}$ и для нахождения $S$ вычислим композицию

$$
W_{*} \circ S=\gamma_{2} \alpha_{0} D_{x}^{2}+\left(R\left(\alpha_{0}\right)+\gamma_{2} \alpha_{1}\right) D_{x}+\sum_{i=0}^{+\infty}\left(W_{*}\left(\alpha_{i}\right)+R\left(\alpha_{i+1}\right)+\gamma_{2} \alpha_{i+2}\right) D_{x}^{-i} .
$$

Согласно построению, описанному в доказательстве леммы $2, W_{*} \circ S=D_{x}^{2}$, и потому $\alpha_{0}=\gamma_{2}^{-1}, \alpha_{1}=-\gamma_{2}^{-1} R\left(\alpha_{0}\right)$, а остальные коэффициенты формального ряда $S$ вычисляются по рекуррентной формуле

$$
\alpha_{i+2}=-\gamma_{2}^{-1}\left(W_{*}\left(\alpha_{i}\right)+R\left(\alpha_{i+1}\right)\right) .
$$


Воспользовавшись этими формулами, получаем

$$
\begin{gathered}
\alpha_{0}=\left(\begin{array}{cc}
p_{x} & 0 \\
0 & q_{x}
\end{array}\right), \quad \alpha_{1}=\left(\begin{array}{cc}
-p_{x x} & \frac{p p_{x} q_{x}}{p q+c} \\
\frac{q q_{x} p_{x}}{p q+c} & -q_{x x}
\end{array}\right), \\
\alpha_{2}=\left(\begin{array}{cc}
p_{x x x}+\frac{p_{x}^{2} q_{x}}{p q+c} & \frac{p_{x}\left(p^{2} q_{x}^{2}-2 c p_{x} q_{x}\right)}{(p q+c)^{2}}-{\frac{p\left(2 p_{x} q_{x x}+q_{x} p_{x x}\right)}{p q+c}}_{p} \\
\frac{q_{x}\left(q^{2} p_{x}^{2}-2 c p_{x} q_{x}\right)}{(p q+c)^{2}}-\frac{q\left(p_{x} q_{x x}+2 q_{x} p_{x x}\right)}{p q+c} & q_{x x x}+{\frac{p_{x} q_{x}^{2}}{p q+c}}^{p}
\end{array}\right) .
\end{gathered}
$$

Как станет ясно из дальнейшего изложения, вычислять остальные члены ряда $S$ в рассматриваемом примере не нужно.

Лемма 2 будет использована для доказательства теоремы следующим образом. Левая часть формулы (13) имеет вид

$$
L \circ S=\left(D_{y}-\vec{F}_{\vec{u}_{x}}\right)\left(\alpha_{0}\right) D_{x}+\sum_{i=0}^{+\infty}\left(L\left(\alpha_{i}\right)+\left(D_{y}-\vec{F}_{\vec{u}_{x}}\right)\left(\alpha_{i+1}\right)\right) D_{x}^{-i}+\cdots,
$$

где многоточием вновь обозначены слагаемые, содержащие ненулевую степень $D_{y}$. Поскольку правая часть (13) не содержит членов с нулевой степенью $D_{y}$, равенство (13) равносильно выполнению цепочки соотношений

$$
\begin{aligned}
\left(D_{y}-\vec{F}_{\vec{u}_{x}}\right)\left(\alpha_{0}\right) & =0, \\
L\left(\alpha_{i}\right)+\left(D_{y}-\vec{F}_{\vec{u}_{x}}\right)\left(\alpha_{i+1}\right) & =0, \quad i=0,1, \ldots
\end{aligned}
$$

Если для некоторого $r$ выполнено $\left(D_{y}-\vec{F}_{\vec{u}_{x}}\right)\left(\alpha_{r+1}\right)=0$, то первые $r+2$ уравнений этой цепочки совпадают с (12). Поэтому теорема была бы доказана, если бы нам удалось модифицировать полученный в лемме 2 ряд $S$ так, чтобы хотя бы один из следующих за $\alpha_{0}$ коэффициентов оказался лежащим в ядре $D_{y}-\vec{F}_{\vec{u}_{x}}$. На достижение этой цели и направлена оставшаяся часть рассуждений. При этом мы будем пользоваться тем, что решение цепочки (15) (если оно существует) не является единственным: для любого формального ряда $M=\sum_{i=0}^{\infty} W_{i} D_{x}^{-i}$ с составленными из $x$-интегралов матричными коэффициентами $W_{i}$ ряды $\widetilde{S}=S \circ M$ и $\widetilde{Q}=Q \circ M$ также будут удовлетворять соотношению (13).

Лемма 3. Пусть система уравнений (2) допускает нетривиальные у-интеграль и найдутся последовательности матрии, $\alpha_{i}, \beta_{i}$ такие, что формальные ряды $S=\sum_{i=0}^{+\infty} \alpha_{i} D_{x}^{-i}$ и $Q=\sum_{i=-1}^{+\infty} \beta_{i} D_{x}^{-i}$ удовлетворяют соотношению (13). Тогда для любого вектора $Z$, составленного из у-интегралов, и всех $i$ выполнены соотношения $Z_{*}\left(\alpha_{i}\right)=0$.

ДокАзАтЕльство. Применяя к вектору $Z$ аналог леммы 1 для $y$-интегралов, получаем

$$
D_{x} \circ Z_{*}=G \circ L
$$

где $G$ - оператор вида $\sum_{j=0}^{m-1} \gamma_{j} D_{y}^{j}, m=\operatorname{ord}_{y}(Z)$. Тогда согласно формуле (13)

$$
D_{x} \circ Z_{*} \circ S=G \circ L \circ S=G \circ Q \circ D_{y} .
$$


Формальный ряд в правой части последнего равенства не содержит членов с нулевой степенью $D_{y}$. Тогда и в левой части равенства все коэффициенты при нулевой степени $D_{y}$ должны равняться нулю. Но, с другой стороны,

$$
D_{x} \circ Z_{*} \circ S=D_{x}\left(\sum_{i=0}^{+\infty} Z_{*}\left(\alpha_{i}\right) D_{x}^{-i}\right)+\cdots,
$$

где опять же многоточием обозначены члены, содержащие ненулевую степень $D_{y}$. Таким образом, $Z_{*}\left(\alpha_{i}\right)=0$ при всех $i$. Лемма 3 доказана.

ЛЕмма 4. Пусть для последовательности матрии, $\alpha_{i}$ размера $n \times n, i=0,1, \ldots$, выполнены соотношения $H\left(\alpha_{i}\right)=0$, где $H=D_{y}^{m}+\sum_{j=0}^{m-1} \gamma_{j} D_{y}^{j} u \gamma_{j}$ - матриць размера $n \times n$. Тогда найдутся натуральное число $r \leqslant n m-\operatorname{rank} \alpha_{0}$ и квадратные матриць $W_{k}, k=0,1, \ldots, r$, такие, что $D_{y}\left(W_{k}\right)=0 u \alpha_{r+1}=\sum_{k=0}^{r} \alpha_{k} W_{k}$.

ДокАзАТЕльство леммы 4 фактически не отличается от доказательства конечномерности над полем констант пространства решений обыкновенного линейного дифференциального уравнения $n$-го порядка. Однако для того чтобы было ясно, как на практике искать матрицы $W_{k}$, мы приведем подробное доказательство.

Для $n$-мерного вектора $\xi$ обозначим через $\xi^{(m)}$ вектор размерности $n m$, у которого первые $n$ компонент совпадают с компонентами вектора $\xi$, следующие $n$ компонент с компонентами вектора $D_{y}(\xi)$, компоненты с $(2 n+1)$-й по $(3 n)$-ю - с компонентами вектора $D_{y}^{2}(\xi)$ и т. д. Для квадратной матрицы $\alpha$ размера $n \times n$ обозначим через $\alpha^{(m)}$ матрицу размера $n m \times n$, столбцы которой получены указанным выше способом из столбцов матрицы $\alpha$. Ясно, что вектор $\xi$ удовлетворяет уравнению $H(\xi)=0$ тогда и только тогда, когда $D_{y}\left(\xi^{(m)}\right)+\Gamma \xi^{(m)}=0$, где через Г обозначена матрица размера $n m \times n m$, образованная из блоков размера $n \times n$ согласно формуле

$$
\Gamma=\left(\begin{array}{cccccc}
0 & -E_{n} & 0 & \ldots & 0 & 0 \\
0 & 0 & -E_{n} & \ddots & 0 & 0 \\
0 & 0 & 0 & \ddots & \ddots & 0 \\
\vdots & \vdots & \vdots & \ddots & \ddots & \vdots \\
0 & 0 & 0 & \ldots & 0 & -E_{n} \\
\gamma_{0} & \gamma_{1} & \gamma_{2} & \ldots & \gamma_{m-2} & \gamma_{m-1}
\end{array}\right)
$$

где $E_{n}$ - единичная матрица размера $n \times n$.

Образуем набор линейно независимых $n m$-мерных векторов $e_{1}, e_{2}, \ldots$, рассматривая по одному столбцы матриц $\alpha_{i}^{(m)}$ и добавляя в этот набор те из столбцов, которые не выражаются в виде линейной комбинации векторов из этого набора. Этот процесс мы будем продолжать до тех пор, пока все столбцы очередной матрицы $\alpha_{r+1}^{(m)}$ не окажутся выражающимися в виде линейной комбинации векторов из набора. Учитывая, что матрица $\alpha_{0}^{(m)}$ содержит не менее rank $\alpha_{0}$ линейно независимых столбцов, а каждая следующая матрица $\alpha_{i}^{(m)}$ должна иметь хотя бы один столбец, не выражающийся через столбцы предыдущих матриц, нетрудно заметить, что описанный выше процесс завершится не позднее, чем $r+\operatorname{rank} \alpha_{0}$ достигнет величины $\mathrm{nm}$. 
Обозначив $i$-й столбец матрицы $\alpha_{r+1}^{(m)}$ через $A_{i}$, получаем

$$
A_{i}=\sum_{j=0}^{l} e_{j} c_{i j}
$$

Но, так как $D_{y}\left(A_{i}\right)+\Gamma A_{i}=0$ для всех $i$ и $D_{y}\left(e_{j}\right)+\Gamma e_{j}=0$ для всех $j$, из $(16)$ вытекает, что

$$
D_{y}\left(A_{i}\right)+\Gamma A_{i}=\sum_{j=0}^{l} e_{j} D_{y}\left(c_{i j}\right)=0
$$

и $D_{y}\left(c_{i j}\right)=0$ в силу линейной независимости векторов $e_{j}$. Учитывая, что $A_{i}$ являются столбцами матрицы $\alpha_{r+1}^{(m)}$ и набор векторов $e_{j}$ образован из столбцов матриц $\alpha_{0}^{(m)}$, $\alpha_{1}^{(m)}, \ldots, \alpha_{r}^{(m)}$, формулы (16) мы можем записать в виде матричного равенства

$$
\alpha_{r+1}^{(m)}=\sum_{k=0}^{r} \alpha_{k}^{(m)} W_{k}
$$

где матрицы $W_{k}$ составлены из $x$-интегралов $c_{i j}$. Для завершения доказательства остается лишь заметить, что из (17) следует $\alpha_{r+1}=\sum_{k=0}^{r} \alpha_{k} W_{k}$. Лемма 4 доказана.

Возвращаясь к рассмотренному выше примеру, заметим, что система (4) допускает $y$-интегралы

$$
z_{1}=\frac{p_{y y}}{p_{y}}-\frac{p q_{y}}{p q+c}, \quad z_{2}=\frac{q_{y y}}{q_{y}}-\frac{q p_{y}}{p q+c} .
$$

Обозначим через $Z$ вектор $\left(z_{1}, z_{2}\right)^{\mathrm{T}}$. В силу леммы 3 найденные нами матрицы (14) удовлетворяют условию $Z_{*}\left(\alpha_{i}\right)=0$. Обозначив через $H$ дифференциальный оператор

$$
\left(\begin{array}{cc}
p_{y} & 0 \\
0 & q_{y}
\end{array}\right) Z_{*},
$$

применим к системе (4) лемму 4. Для нахождения матриц $W_{k}$ возьмем в качестве $e_{1}$ и $e_{2}$ первый и второй столбцы матрицы $\alpha_{0}^{(2)}$, а в качестве $e_{3}-$ первый столбец матрицы $\alpha_{1}^{(2)}$. Столбцы матрицы $\alpha_{2}^{(2)}$ лежат в линейной оболочке векторов $e_{1}, e_{2}$ и $e_{3}$. Выразив столбцы матрицы $\alpha_{2}^{(2)}$ через $e_{1}, e_{2}$ и $e_{3}$, получаем

$$
\alpha_{2}=\alpha_{0} W_{0}+\alpha_{1} W_{1}
$$

где

$$
\begin{aligned}
W_{1} & =\left(\begin{array}{cc}
\frac{2 q_{x} p+q p_{x}}{p q+c}-\frac{q_{x x}}{q_{x}}-2 \frac{p_{x x}}{p_{x}} & 2 \frac{q_{x x}}{q_{x}}+\frac{p_{x x}}{p_{x}}-\frac{q_{x} p+2 q p_{x}}{p q+c} \\
0 & 0
\end{array}\right), \\
W_{0} & =\left(\begin{array}{ll}
w_{11} & w_{12} \\
w_{21} & w_{22}
\end{array}\right),
\end{aligned}
$$




$$
\begin{aligned}
& w_{11}=\frac{p_{x x x}}{p_{x}}-\frac{p_{x x} q_{x x}}{q_{x} p_{x}}-2 \frac{p_{x x}^{2}}{p_{x}^{2}}+\frac{2 q_{x} p p_{x x}}{(p q+c) p_{x}}+\frac{q p_{x x}+p_{x} q_{x}}{p q+c}, \quad w_{21}=\frac{-2 p_{x} q_{x}}{p q+c}, \\
& w_{12}=2 \frac{p_{x x} q_{x x}}{p_{x} q_{x}}-2 \frac{q_{x x} p+q p_{x x}}{p q+c}+\frac{p_{x x}^{2}}{p_{x}^{2}}-\frac{2 q_{x} p p_{x x}}{p_{x}(p q+c)}+\frac{q_{x}^{2} p^{2}-2 p_{x} q_{x} c}{(p q+c)^{2}}, \\
& w_{22}=\frac{q_{x x x}}{q_{x}}-\frac{2 q p_{x} q_{x x}}{q_{x}(p q+c)}+\frac{p_{x} q_{x}-q p_{x x}}{p q+c}+\frac{q p_{x}\left(2 q p_{x}+p q_{x}\right)}{(p q+c)^{2}} .
\end{aligned}
$$

Непосредственным вычислением можно проверить, что $D_{y}\left(W_{0}\right)=0$ и $D_{y}\left(W_{1}\right)=0$, как это и следует из леммы 4.

ДОКАЗАТЕЛЬСТво ТЕОРЕмЫ. В силу леммы 2 найдутся формальные ряды $\widehat{S}=$ $\sum_{i=0}^{+\infty} \hat{\alpha}_{i} D_{x}^{-i}$ и $\widehat{Q}=\sum_{i=-1}^{+\infty} \hat{\beta}_{i} D_{x}^{-i}$ такие, что выполнено соотношение $L \circ \widehat{S}=\widehat{Q} \circ D_{y}$. В силу леммы 3 выполнены равенства $Z_{*}\left(\hat{\alpha}_{i}\right)=0$. Переписав эти равенства в виде $\left(Z_{\vec{v}_{m}}\right)^{-1} Z_{*}\left(\hat{\alpha}_{i}\right)=0$, мы можем применить лемму 4 и получить, что для некоторого $r \leqslant n m-\operatorname{rank} \alpha_{0}=n m-n$ выполнено равенство $\hat{\alpha}_{r+1}=\sum_{i=0}^{r} \hat{\alpha}_{i} W_{i}$, где $W_{i}-$ квадратные матрицы такие, что $D_{y}\left(W_{i}\right)=0$.

Нетрудно видеть, что для любой квадратной матрицы $W$ такой, что $D_{y}(W)=0$, и любого $j$ верно равенство

$$
L \circ \widehat{S} \circ W \circ D_{x}^{-j}=\widehat{Q} \circ W \circ D_{x}^{-j} \circ D_{y} .
$$

В силу этого формальные ряды $S_{1}=\widehat{S}-\widehat{S} \circ W_{r} \circ D_{x}^{-1}$ и $Q_{1}=\widehat{Q}-\widehat{Q} \circ W_{r} \circ$ $D_{x}^{-1}$ также удовлетворяют соотношению $L \circ S_{1}=Q_{1} \circ D_{y}$. Воспользовавшись формулой (10), получаем, что коэффициент при $D_{x}^{-(r+1)}$ в $\widehat{S} \circ W_{r} \circ D_{x}^{-1}$ равен $\hat{\alpha}_{r} W_{r}+\sum_{i=1}^{r-1} C_{-i}^{r-i} \hat{\alpha}_{i} D_{x}^{r-i}\left(W_{r}\right)$. Поэтому коэффициент при $D_{x}^{-(r+1)}$ в $S_{1}$ равен $\hat{\alpha}_{0} W_{0}+$ $\sum_{i=1}^{r-1} \hat{\alpha}_{i} W_{i, 1}$, где $W_{i, 1}=W_{i}-C_{-i}^{r-i} D_{x}^{r-i}\left(W_{r}\right)$. Вычитая из $S_{1}$ формальный ряд $\widehat{S} \circ W_{r-1,1} \circ D_{x}^{-2}$, получим формальный ряд $S_{2}$, у которого коэффициент при $D_{x}^{-(r+1)}$ равен $\hat{\alpha}_{0} W_{0}+\sum_{i=1}^{r-2} \hat{\alpha}_{i} W_{i, 2}$, причем $D_{y}\left(W_{i, 2}\right)=0$, и т.д. Повторив описанную выше процедуру несколько раз, мы придем к формальному ряду $S_{r}=\sum_{i=0}^{+\infty} \alpha_{i} D_{x}^{-i}$ такому, что $L \circ S_{r}=Q_{r} \circ D_{y}$, причем матрица $\alpha_{0}=\hat{\alpha}_{0}$ невырожденна и $\alpha_{r+1}=\hat{\alpha}_{0} W_{0}$. Остается заметить, что коэффициенты $\alpha_{i}$ построенного нами ряда $S_{r}$ удовлетворяют цепочке соотношений $(15)$, первые $r+2$ из которых в силу равенства $\left(D_{y}-\vec{F}_{\vec{u}_{x}}\right)\left(\alpha_{r+1}\right)=0$ переходят в уравнения (12). Теорема доказана.

Воспользовавшись проведенными ранее вычислениями и следуя доказательству теоремы, построим формулу для симметрий системы (4). С учетом формулы (18) для этого нам надо вычислить

$$
S_{1}=S-S \circ W_{1} \circ D_{x}^{-1}=\alpha_{0}+\left(\alpha_{1}-\alpha_{0} W_{1}\right) D_{x}^{-1}+\alpha_{0} W_{0} D_{x}^{-2}+\cdots,
$$

где $\alpha_{0}$ и $\alpha_{1}$ задаются формулами (14), а $W_{1}$ - формулой (19). Так как $\left(D_{y}-\right.$ $\left.\vec{F}_{\vec{u}_{x}}\right)\left(\alpha_{0} W_{0}\right)=0$, коэффициенты при $D_{x}^{0}$ и $D_{x}^{-1}$ в ряде $S_{1}$ удовлетворяют цепочке соотношений (12) с $r=1$. Таким образом, подставив найденные ранее значения $\alpha_{0}$, $\alpha_{1}$ и $W_{1}$ в $\alpha_{0} D_{x}+\left(\alpha_{1}-\alpha_{0} W_{1}\right)$, получаем, что дифференциальный оператор

$$
\left(\begin{array}{cc}
p_{x} & 0 \\
0 & q_{x}
\end{array}\right) D_{x}+\left(\begin{array}{cc}
p_{x x}+\frac{p_{x} q_{x x}}{q_{x}}-\frac{2 p p_{x} q_{x}+q p_{x}^{2}}{p q+c} & 2 \frac{p p_{x} q_{x}+q p_{x}^{2}}{p q+c}-2 \frac{p_{x} q_{x x}}{q_{x}}-p_{x x} \\
\frac{q q_{x} p_{x}}{p q+c} & -q_{x x}
\end{array}\right)
$$


переводит любой двумерный вектор, составленный из $x$-интегралов, в вектор симметрий системы (4).

Заметим, что как в приведенном выше примере, так и в других примерах порядок $r$ оператора, переводящего интегралы в симметрии, удовлетворяет неравенству $r<m$, где $m$ - порядок набора из $n$ независимых в главном интегралов. Это позволяет предположить, что полученная в теореме оценка для $r$ является весьма грубой.

Благодарности. “Авторы” благодарны А. В. Жиберу и А. Б. Шабату за полезные обсуждения. Работа выполнена при финансовой поддержке РФФИ (гранты № 05-01-00775-а (В. В. Соколов) и № 07-01-00081-а (С. Я. Старцев)).

\section{Список литературы}

[1] А. В. Жибер, А. Б. Шабат, Докл. АН СССР, 247:5 (1979), 1103-1107.

[2] А. В. Жибер, В. В. Соколов, УМН, 56:1(337) (2001), 63-106.

[3] Ф. Трикоми, Лекиии по уравнениям в частных производных, ИЛ, М., 1957.

[4] A. N. Leznov, M. V. Saveliev, Lett. Math. Phys., 3:6 (1979), 489-494.

[5] А. Н. Лезнов, ТМФ, 42:3 (1980), 343-349.

[6] А.Б. Шабат, Р. И. Ямилов, Экспотенииалъные системы типа I и матрищы Картана, Препринт, Башкирский филиал АН СССР, Уфа, 1981.

[7] A. B. Shabat, Phys. Lett. A, 200:2 (1995), 121-133.

[8] А. Н. Лезнов, В. Г. Смирнов, А. Б. Шабат, ТМФ, 51:1 (1982), 10-22.

[9] А.Н. Лезнов, А. Б. Шабат, "Условия обрыва рядов теории возмущений”, Интегрируемые системы, Башкирский филиал АН СССР, Уфа, 1982, 34-45.

[10] Д. К. Демской, С. Я. Старцев, Фундамент. и прикл. матем., 10:1 (2004), 29-37.

[11] С. Я. Старцев, Фундамент. и прикл. матем., 12:7 (2006), 251-262. 